\title{
TIPOS DE EVALUACIONES EDUCATIVAS APLICADAS A ESTUDIANTES DE HISTORIA, URACCAN, NUEVA GUINEA
}

Blanca Nevai Centeno Bravo ${ }^{[19]}$

\section{Resumen}

El estudio se realizó en el municipio de Nueva Guinea con el propósito de obtener una visión holística de la realidad evaluativa que el personal docente aplica en el proceso de enseñanza en la carrera Historia. La población en estudio fueron 29 estudiantes y 2 docentes.

Los Tipos de evaluaciones educativas aplicadas en Ciencias de la Educación con mención en Historia encuentros bimestrales, Recinto URACCAN, Nueva Guinea, RAAS, primer semestre 2008, tiene un enfoque cualitativo etnográfico y centró su interés en conocer las diferentes formas de evaluación que fueron aplicados en el proceso enseñanza aprendizaje.

Las unidades de análisis fueron los estudiantes, los docentes, y las fuentes documentales (syllabus, programas de estudio, y planes de clase). Los instrumentos de recolección de información estuvieron basados en la observación directa en el aula de clase, la entrevista a los docentes y un grupo focal con los educandos.

Las conclusiones más relevantes respecto a los tipos de evaluaciones utilizados en el proceso enseñanza-aprendizaje demostró que todavía se aplican exámenes escritos como forma taxativa de evaluación de la enseñanza.

Los docentes construyen con los educandos la metodología evaluativa, logrando de esa manera que los estudiantes sean también parte activa dentro del proceso enseñanza aprendizaje.

Palabras claves: Aprendizaje, docente, examen, evaluación, estudiantes, enseñanza, proceso.

\section{Introducción}

La evaluación del aprendizaje es un proceso sistemático que comprende la búsqueda y obtención de información de diversas fuentes acerca de la calidad del desempeño,

[19] Máster en docencia universitaria, docente de URACCAN. blancanevai@gmail.com 
avance, rendimiento o logro del estudiante y de la calidad de los procesos empleados por el docente.

El objetivo de cualquier proceso de enseñanza, es conseguir que cada estudiante aprenda de forma significativa para la vida, pero a lo largo de este proceso se encuentran con obstáculos y dificultades ante los cuales los docentes deberán identificar cuáles son estos obstáculos y superarlos adecuadamente, de ahí la necesidad de una evaluación sistemática.

En el Recinto de Nueva Guinea las evaluaciones se efectúan mediante dos exámenes parciales con un valor de 60 y el 40 por ciento respectivamente, lo cual acumulan en diversas actividades en donde el estudiante realiza exposiciones, seminarios, investigaciones bibliográficas entre otras formas; en algunas ocasiones el docente puede variar la evaluación y se decide en conjunto con los estudiantes. Las estrategias de evaluación como característica de la asignatura, estarán enfocadas en menor cantidad al uso de exámenes escritos; sino más bien a trabajos de exposición oral, trabajos escritos, ubicación en el mapa, informe de investigación bibliográfica, discusiones, debates y en especial la participación individual y grupal.

Con el presente trabajo de los tipos de evaluación educativas aplicadas en estudiantes de la carrera de licenciatura en Ciencias de la Educación con mención en Historia, durante el primer semestre del año 2008, URACCAN, Recinto Nueva Guinea; se describen los instrumentos aplicados a la evaluación durante el proceso de aprendizaje de los estudiantes; así como la percepción del estudiante en cuanto a los tipos de evaluación educativa aplicadas y la idoneidad de los diferentes tipos de instrumentos aplicados en el proceso evaluativo del aprendizaje

\section{Revisión de Literatura}

Según Roncal (2005) en el año 1990, el pedagogo Popham analiza el concepto sobre evaluación, se puede decir que es una acción inherente a toda actividad humana, la cual debe ser sistemática, y que su objetivo es determinar el valor de algo.

La educación es una práctica social y la evaluación uno de sus principales actos que se lleva a cabo en las instituciones educativas y debemos abordarla desde distintos aspectos: ideológicos, sociales, pedagógicos, psicológicos y técnicos (Gorritti, 2006).

En 2005, Roncal valorará la evaluación como un juicio educativo y calificativo que se da sobre una persona o situación basándose en una evidencia constatable.

Según el modelo Pedagógico de la URACCAN (2004) se concibe la evaluación como: proceso continuo, considera las diferencias individuales, apunta al desarrollo 
de habilidades, integral, toma en cuenta el desempeño, permite la reflexión de los estudiantes, es participativo y crítico.

La URACCAN establece en el Reglamento de trabajo científico estudiantil (2004) artículo No 4. Que la evaluación del aprendizaje no debe centrarse únicamente alrededor de los conocimientos requeridos para las certificaciones de habilidades, ni debe basarse en un modelo único que se aplique en todos los contextos.

La evaluación del aprendizaje se puede realizar mediante un trabajo científicoestudiantil, el cual consiste en las actividades que realizan los estudiantes en forma de sistema durante el proceso enseñanza-aprendizaje, siendo su objetivo primordial contribuir al desarrollo de habilidades y hábitos propios del trabajo técnico y científicoinvestigativo (Reglamento estudiantil, 2004).

\section{Materiales y Métodos}

Las fuentes primarias de investigación fueron: el grupo focal aplicado a los estudiantes con mención en historia, las entrevistas dirigidas a los docentes que impartían clases, observaciones directas a pie de aula durante el proceso educativo y la revisión documental en lo que se refiere a programas, syllabus, planes de clase en la carrera de Ciencias de la Educación.

Una vez obtenidos los datos requeridos, se procedió al análisis de la información, afín de llegar a las conclusiones y plantear algunas recomendaciones.

\section{Resultados y Discusión}

A través de los instrumentos aplicados tanto a docentes como a estudiantes, se constató que la evaluación más usual es el examen acompañado de otros trabajos prácticos en el VII semestre, se cumple con los tres momentos de la evaluación: la evaluación diagnóstica, partiendo de los conocimientos previos que poseen los estudiantes. Esto permitió que los docentes estuvieran al tanto del nivel de conocimiento que posee cada estudiante; la evaluación sumativa permitió verificar el logro de objetivos o competencias que determinaban una nota (cuantitativa) de aprobado o reprobado en el curso; y, por último, la evaluación formativa que le permitió a los docentes incidir de forma pertinente en la formación de ciudadanía crítica, participativa y analítica.

En la información obtenida mediante el grupo focal, los estudiantes expresaron que los docentes realizaron diferentes formas de evaluación como la sumativa, cualitativa, cuantitativa y, con anterioridad, la evaluación diagnóstica. La evaluación varía al utilizarse diferentes modos o formas. 
Estas actividades evaluativas en donde intervienen exclusivamente los educandos de manera activa, como por ejemplo: 1) exposiciones orales a partir de guías prediseñadas por lo que la y él docente ha orientado; 2) documentos de apoyo en la que se han planteado situaciones donde con posterioridad se ejecuta la evaluación sumativa; 3) trabajos investigativos donde los docentes asigna una situación para indagar problemáticas y posibles conclusiones; 4) ensayos prácticos donde los estudiantes sitúa los conocimientos adquiridos en el contexto de su realidad formativa; 5) el trabajo de laboratorio, que consistió en trabajos prácticos en grupo e individual donde se especificaban las acciones a realizar durante la clase; y por último 6) el examen escrito.

Tradicionalmente se han denominado sistemáticos a aquellas pruebas que se ejecutan cuando el número de objetivos a evaluarse es relativamente correspondiente al número de contenidos impartidos, para luego sumarse con el examen escrito orientado a las unidades programáticas estipuladas en los syllabus.

Estas características que presenta el examen escrito, son los que en resumidas cuentas, dan el resultado o "calificación" que se le asigna a cada estudiante. Sin embargo, deberá tomarse en cuenta que esta puntuación cuantitativa no revela el nivel de aprendizaje de los discentes.

Lo anterior expuesto, se contrapone con el enfoque constructivista de la enseñanza educativa, pues éste en alguna medida invoca a la evaluación no como una recolección de números donde pueda encasillarse el conocimiento de los educandos, cual si fuera un recipiente de conocimientos. Pero, por las condiciones de la URACCAN, y el reglamento de esta institución donde especifica que deberá ponerse una cifra de o a 100, el área de Registro Académico exige que los docentes coloquen en sus actas de calificaciones números. De ahí que, el Reglamento de Registro Académico de URACCAN, aprobado en sesión ordinaria número 3 (2005), reza en su artículo Art. 80:

La calificación final en cada asignatura se obtendrá de acuerdo al sistema de evaluación de la siguiente forma: primera evaluación 50 por ciento, segunda evaluación 50 por ciento. Se exceptúan de esta disposición: a) aquellas asignaturas que por su naturaleza, concluyan con trabajo de cursos, cuya calificación final será la nota del curso; b) asignaturas cursadas en modalidad por encuentros intensivos. Todas las calificaciones se expresarán en la escala de o a 100 puntos, siendo 60 la nota mínima para aprobar. (p. 12)

A través de la revisión documental efectuada, los materiales consultados como los programas, se constató que la forma que está planteada la evaluación es significativamente importante el examen escrito. El primer examen al finalizar la III Unidad con un valor de 50 por ciento con trabajos como seminarios, clases prácticas, trabajos de investigación, exposiciones, etc. El mismo procedimiento se realizará con el segundo parcial evaluando hasta la VI unidad del programa, posteriormente se sumarán las dos 
calificaciones y se dividen entre dos obteniendo la nota final. Los programas tienen una carga horaria 48 horas clases. En sus syllabus están presentes los tres momentos de la evaluación diagnóstica, sumativa y formativa. De forma más especifica presento el cuadro evaluativo del programa de la asignatura de Introducción a la Informática, correspondiente al VII semestre de la carrera.

Cuadro No. 1. Programa evaluativo de la asignatura Introducción a la Informática VII semestre de la carrera.

\begin{tabular}{lllll}
\hline \multirow{2}{*}{ Parciales } & \multicolumn{2}{c}{ Distribución del tiempo } & \multicolumn{2}{c}{ Distribución del tiempo } \\
\cline { 2 - 5 } & Exámenes & Proceso & C.T & Clases teóricas \\
\hline Primer Parcial & 50 & 50 & T.P & Técnico Práctico \\
\hline Segundo Parcial & 50 & 50 & P.P & Pruebas Parciales \\
\hline
\end{tabular}

La nota Final es el promedio de los dos exámenes parciales.

En los hallazgos sobre los planes de clase, en lo que corresponde a la evaluación de los aprendizajes, se constató que los docentes desarrollan los tres momentos evaluativos, realizan actividades como la evaluación de los conocimientos previos de los estudiantes; explica con sus palabras lo que entienden por hoja de cálculo Excel, tipos de datos, el procedimiento de forma sencilla para crear una nueva hoja, un nuevo libro, introducir fórmulas en Microsoft Excel, destacando el orden en que se debe realizar y evalúa a los estudiantes de forma cuantitativa y cualitativa. El sistema de evaluación encontrado en el syllabus es el siguiente:

- Examen parcial . . . . . . . . . . . . 50\%

- Pruebas cortas . . . . . . . . . . . . 10\%

- Participación individual y practica de valores . . . .10\%

- Trabajo de campo y presentación. . . . . . . . .40\%

En la aplicación del instrumento de observación docente en el aula de clase, durante el desarrollo del proceso de enseñanza-aprendizaje logramos encontrar diferentes aspectos que tiene su incidencia en la aplicación de los tipos de evaluación empleado con los estudiantes del VIII semestre de la carrera de licenciatura en Ciencias de la Educación con mención en Historia. El instrumento diseñado para la observación docente directa, está planteado con ciertos aspectos de valoración desde, aspectos técnicos y didácticos empleados y los tipos de evaluación aplicadas durante el proceso de enseñanza-aprendizaje hasta las cualidades personales del docente. 
En cuanto a los aspectos técnicos-didácticos, se contaba con un plan de clase, objetivo, contenido y aprendizaje; además con una muy buena organización del aprendizaje; el desarrollo de la clase se da con el inicio de la relación del tema anterior en conexión del tema a enseñar, con la orientación de los objetivos, realizando ejemplos adecuados para la clase, al darle cumplimiento a los objetivos y actividades del proceso.

Se da la retroalimentación del contenido a impartir la evaluación diagnóstica como uno de los componentes de la evaluación, permitiendo de esa manera la combinación de la enseñanza y la educación, más cuando se da la atención de forma individual a los estudiantes, permitiendo de esa manera la interacción con los educandos, hay una correcta organización del salón de clase, que permite la conducción del aprendizaje.

En la parte de los aspectos técnicos-didácticos, alcanzamos observar el buen uso de los medios audiovisuales, que le da a los docentes la oportunidad de una clase bien motivada, más aún cuando se vincula la práctica con la teoría, logrando de esa forma que el educando participe activamente, promoviendo una política de participación y diálogo entre el docente y discente.

Saber cómo enseñar ciencias es, lógicamente, uno los cometidos de la enseñanza de las Ciencias Sociales. Los docentes, quienes juegan un papel importante en el proceso de enseñanza aprendizaje de las y los discentes, manifestaron que ellos realizaban trabajos prácticos, tareas e investigación. No realizan exámenes parciales, aunque no todos expresaron la no realización de exámenes, debido a que aún consideran que el examen se debe de utilizar como un instrumento de medición.

Durante la observación docente se identificó que la parte práctica es de gran importancia en el aprendizaje, sobre todo cuando se trata de una asignatura que requiere de bastante actividad práctica. En ciertas ocasiones los estudiantes acumulan su nota cuantitativa mediante la realización de ejercicios prácticos, dependiendo del grupo de estudiante y las habilidades que tengan y vayan desarrollando sobre todo cuando es una asignatura nueva para ellos, se cita el caso en este grupo de la Introducción a la Informática, en la cual se observó, grandes dificultades por parte de los discentes.

Los educandos no participan en la elaboración de los instrumentos; pero, en algunas situaciones si hacen sugerencias al docente de la forma de cómo evaluar su aprendizaje, en estos dos últimos semestres aplicaron la forma de evaluación de algunos docentes de no realizar exámenes escritos, sino que ellos acumularan y funcionó de forma muy significativa para su aprendizaje.

EL Modelo Pedagógico de la URACCAN (2004), describe la evaluación del aprendizaje como un proceso continuo que debe acompañar al proceso de enseñanza ya que ambos son inseparables (p.19). De ahí que, el modelo pedagógico de la URACCAN, 


\section{EDUCACIÓN}

aprobado en sesión ordinaria marzo 2004 del Consejo Universitario, cuya reforma del 6 al 8 de Septiembre 2004, dice textualmente:

El mismo define la evaluación del aprendizaje como: Un proceso sistemático y permanente que comprende la búsqueda y obtención de información de diversas fuentes acerca de la calidad del desempeño, avance, rendimiento o logro del estudiante y de la calidad de los procesos empleados por el docente, la determinación de su importancia y pertinencia de conformidad con los objetivos de formación que se espera alcanzar, todo con el fin de tomar decisiones que orienten el aprendizaje y los esfuerzos de la gestión docente. (p. 20)

El Reglamento de trabajo científico estudiantil determina que la evaluación del aprendizaje no debe centrarse únicamente alrededor de los conocimientos requeridos para las certificaciones de habilidades, ni debe basarse en un modelo único que se aplique en todos los contextos.

Puede ser mediante el trabajo científico-estudiantil, el cual consiste en las actividades que realizan los estudiantes en forma de sistemático durante el proceso enseñanza-aprendizaje, siendo su objetivo primordial contribuir al desarrollo de habilidades y hábitos propios del trabajo técnico y científico-investigativo, incorporando en forma creciente y progresiva, la búsqueda de soluciones a problemas no resueltos con los conocimientos actuales e identificando disyuntivas para enfrentar problemas reales, o valore el impacto de los ya resueltos (URACCAN; 2004).

El Reglamento de trabajo científico estudiantil (2004) en su artículo No 4., considera que los instrumentos o trabajos de fin curso se definen de acuerdo a objetivos de los planes de estudio y programas de asignaturas, desarrollándose en el semestre en que se imparte la asignatura.

El reglamento de trabajos de fin de curso, URACCAN (2004), textualmente en su artículo No. 9. reza:

El colectivo de estudiantes, presentará un informe del trabajo de curso realizado en forma mecanografiada o digitalizada. El trabajo deberá contener como mínimo los aspectos siguientes: Introducción, antecedentes, justificación, objetivos, marco teórico, referencial o conceptual, metodología, resultados, conclusiones y recomendaciones, lista de referencias bibliográficas y anexos (p.3).

Teniendo en cuenta lo anterior, se puede decir, que no solamente el examen es el único instrumento con el cual los docentes pueden evaluar a sus estudiantes durante el proceso de aprendizaje. 
A través del grupo focal se comprobó que los estudiantes prefieren ser evaluados con instrumentos pedagógicos donde ellos sean los principales partícipes en el proceso enseñanza-aprendizaje como: el seminario, trabajos prácticos, guías de laboratorio, investigaciones. De esta forma, expresan sentirse mejor porque creen que los aprendizajes son adquiridos de formas más adecuadas y lograr un aprendizaje significativo en su formación como docentes en práctica.

\section{Conclusiones}

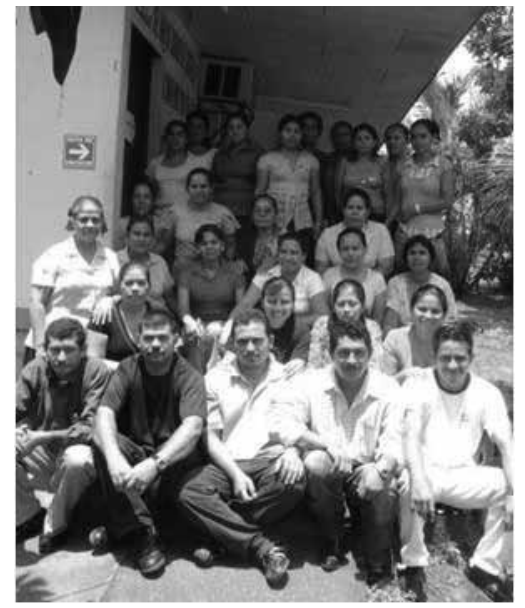

La evaluación de los aprendizajes reconoce como ámbito o espacio típico aquello que sucede en el aula de clase, demanda estrategias metodológicas precisas destinadas a registrar indicios claros sobre un proceso tan complejo e individual como la evaluación del aprendizaje.

El resultado investigativo determina que los tipos de evaluación aplicados en el proceso educativo por los docentes se basan en un examen escrito, se presentan los tres momentos de la evaluación: diagnóstica, sumativa y formativa. Los trabajos prácticos aplicados en la evaluación del aprendizaje tienen los requisitos que debe contener un instrumento educativo y evaluativo como es la parte cuantitativa y la cualitativa. La redacción de ensayos como un instrumento de evaluación del aprendizaje, permite el desarrollo de habilidades en los estudiantes.

Los docentes consideran que el examen escrito es un instrumento viable para determinar el conocimiento del educando y en ciertas ocasiones se utiliza como un instrumento evaluador en donde lo único que deseamos es demostrar que no es el estudiante el que sabe, sino el docente. La mayoría de las ocasiones los instrumentos de evaluación aplicados a los discentes no cumplen con los parámetros requeridos de pruebas escritas.

Sin duda, muchos/as docentes no recurren a otros tipos de instrumentos de evaluación necesarios para una valoración de procesos del/la educando/a.

La valoración cuantitativa que promueve URACCAN, no corresponde con un enfoque constructivista de la educación, pues, se deslinda de la lectura de Registro Académico, de la forma de evaluación está dirigida a la cuantificación del aprendizaje de los/las aprendices. 


\section{EDUCACIÓN}

La evaluación de los aprendizajes reconoce como ámbito o espacio típico aquello que sucede en el aula, demanda estrategias metodológicas precisas destinadas a registrar indicios claros sobre un proceso tan complejo e individual como la evaluación del aprendizaje.

\section{Conclusiones:}

- Los tipos de evaluación aplicados en el proceso enseñanza aprendizaje por los docentes se basan en examen escrito.

- Se aplican los tres momentos de la evaluación: diagnóstica, sumativa y formativa.

- En la asignatura de Introducción a la Informática, se realizaron trabajos prácticos con una guía de estudio y actividades pedagógicas en el laboratorio.

- Los trabajos prácticos aplicados en la evaluación del aprendizaje tienen los requisitos que debe contener un instrumento educativo y evaluativo como es la parte cuantitativa y la cualitativa.

- La redacción de ensayos como un instrumento de evaluación del aprendizaje, permite el desarrollo de habilidades en los estudiantes.

- Los docentes consideran que el examen escrito es un instrumento viable para determinar el conocimiento del educando.

- La mayoría de las ocasiones los instrumentos de evaluación aplicados a los discentes no cumplen con los parámetros requeridos de pruebas escritas.

- Sin duda, muchos docentes no recurren a otros tipos de instrumentos de evaluación necesarios para una valoración de procesos del educando.

- La valoración cuantitativa que promueve URACCAN, no corresponde con un enfoque constructivista de la educación, pues, se deslinda de la lectura de Registro Académico, que la forma de evaluación está dirigida a la cuantificación del aprendizaje.

Teniendo en cuenta que el proceso de evaluación es un requisito imprescindible de todo proceso formativo-educativo, y que la URACCAN en su misión como Institución encargada de velar por el desempeño de los docentes y de sus aprendices, considera importante como parte de su misión la formación de sus recursos humanos, con conocimientos y capacidades científico-técnicas, y en el pleno goce de ese derecho, comprende que la evaluación que no contribuya a aprender de modo más cualificado y que no contribuya a fortalecer el aprendizaje de los estudiantes en los diferentes niveles educativos es mejor no practicarla. 


\section{Por cuanto las recomendaciones que se plantean en torno al estudio, se deberán tomar las siguientes:}

- Diseñar otras estrategias de evaluación que permitan la participación activa tanto del docente como del discente en función de los aprendizajes.

- Que las Áreas Académicas del Recinto de Nueva Guinea, continúen diseñando cuando menos dos veces al año, propuestas de capacitaciones encaminadas a la elaboración de nuevas formas de evaluación que permitan el acceso y aprendizaje de los docentes para su posterior aplicación.

- Realizar un plan de acompañamiento a los docentes que consideran aún el uso de los exámenes o pruebas escritas como único recurso didáctico de evaluación.

- Todo proceso formativo deberá tener un proceso evaluativo que registre los progresos y dificultades que se va teniendo en la ejecución de los programas de clases.

- Recurrir a otras formas de evaluación siempre será una salida viable al quehacer educativo, por lo que su aplicación deberá estar contenida en los programas y syllabus, y ser planificadas con las apropiadas orientaciones de objetivos.

- Continuar con la revisión curricular de los planes de estudio y mejorar la parte correspondiente a la evaluación del aprendizaje.

\section{Lista de Referencias}

Carballo, R. \& Bordón (1990). Evolución del concepto de evaluación: desarrollo de los modelos de evaluación de Programas.

\section{Carmen Leticia Gorritti Siappo (2005). Evaluación Educativa.}

Roncal Martínez, Federico (2005). Evaluación del Aprendizaje. La Salle, Guatemala.

Roncal Martínez, Federico (2006). Educación Intercultural. La Salle, Guatemala.

URACCAN (2004). Documentos normativos de los trabajos de graduación: Reglamento del trabajo científico estudiantil y de la culminación de los estudios. Capitulo No 1, arto.1.p.2. 